ECCOMAS

Proceedia
COMPDYN 2021

$8^{\text {th }}$ ECCOMAS Thematic Conference on Computational Methods in Structural Dynamics and Earthquake Engineering M. Papadrakakis, M. Fragiadakis (eds.) Streamed from Athens, Greece, 28 - 30 June 2021

\title{
LIFE-CYCLE COST ANALYSIS OF TALL BUILDINGS IN SYNOPTIC, MIXED WIND LOAD CLIMATES BY LAYERED STOCHASTIC APPROXIMATION MONTE-CARLO METHOD
}

\author{
Lei Zhang ${ }^{1}$, Luca Caracoglia ${ }^{2}$ \\ ${ }^{1} \mathrm{PhD}$ Candidate, Dept. of Civil and Environmental Engineering, Northeastern University \\ Boston, MA 02115, USA \\ e-mail: zhang.lei1@ northeastern.edu \\ ${ }^{2}$ Associate Professor, Dept. of Civil and Environmental Engineering, Northeastern University \\ Boston, MA 02115, USA \\ e-mail: lucac@coe.neu.edu
}

\begin{abstract}
This paper extends the existing formulation for life-cycle cost analysis (LCCA) of tall buildings subjected to synoptic, mixed wind load climates. This situation arises, for example, if the site is characterized by the presence of both tropical cyclones and extra-tropical depressions. A parametric study is conducted to examine the dependence of building's intervention cost, due to wind-induced damage, on the building height. Special attention is paid to the influence of wind load directionality, enabled by the modeling of the joint probability of mean-wind speed and direction using an angular-linear model. Furthermore, the Layered Stochastic Approximation Monte-Carlo (LSAMC) method is implemented for fragility analysis, as an alternate, surrogate approach compared to the standard Monte-Carlo simulation via physical building model. The results of the LSAMC simulations are validated by Monte-Carlo sampling. The study demonstrates that the LSAMC method is eligible for wind-induced fragility analysis while simultaneously achieving a remarkable reduction in computing time.
\end{abstract}

Keywords: Mixed Wind Load Climates; Layered Stochastic Approximation Monte-Carlo Method; Wind Directionality; Life-cycle Cost Analysis. 


\section{INTRODUCTION}

Tall buildings tend to oscillate in the along-wind, across-wind and torsional directions under the effect of dynamic wind loads, arising from variable pressure distribution on the building surface [1]. Due to the aleatory nature of the wind flow, efficiently quantifying wind loads can be problematic for structural design. Great efforts have been devoted to the estimation of wind load effects in the presence of uncertainties [2, 3, 4]. Adapted from performancebased design that was introduced for seismic engineering, the field of performance-based wind engineering (PBWE) has been introduced as an efficient approach that accounts for various uncertainties, inherent in the interactions between structures and wind loads. PBWE has been implemented for a wide range of structures and extreme wind phenomena; examples include low-rise, light-frame wood structures subjected to hurricanes [5], tall buildings [6, 7, 8], longspan bridges $[9,10]$, and various structures affected by non-stationary wind loads (e.g. tornadoes, thunderstorms) [11].

At the core of the PBWE is the uncertainty evaluation and fragility determination. Uncertainty sources may incorporate wind turbulence, structural deterioration and experimental conditions, used for wind load estimation. Some of these factors are aleatory, e.g. wind turbulence, and thus cannot be eliminated, while uncertainties related to experiments can be minimized by improving testing facilities and protocols. The concept of "fragility" is used to examine likelihood of failure occurrence contingent on the prescribed uncertainties. In practice, the fragility is expressed as the exceedance probability of a predefined performance objective, conditional on a variable (e.g. mean wind speed) describing the intensity of the demand on the structure. Performance objectives are described as limit-state conditions by a resultant structural effect, for example, in tall buildings the top-floor lateral drift and acceleration for a serviceability limit state and the internal stresses in a corner column for an ultimate limit state.

Currently, Monte-Carlo simulation is widely used for the assessment of fragility because it is applicable to any structural, physical model and it is simple to implement. However, an entrenched problem that the Monte-Carlo-based approach faces is that the process requires the generation of a large number of realizations and, consequently, is time consuming and computationally expensive. To overcome this limitation, attempts have been made by using sophisticated techniques [12] and improved algorithms, e.g. the Layered Stochastic Approximation MonteCarlo (LSAMC) method [13]. The LSAMC method is based on the Stochastic Approximation algorithm [14]; it can derive the statistics of the performance objective indicator, expediting the procedure for fragility evaluation while preserving fidelity. The objectives of this paper are to: (i) describe the results of a parametric study implementing LSAMC-based fragility analysis for a benchmark tall building with variable heights and (ii) investigate the dependency of life-cycle cost assessment on the building height within the PBWE framework [15].

\section{BACKGROUND THEORY}

\subsection{Multi-directional aerodynamic response of tall buildings}

Capitalizing on the frequency-domain random vibration analysis for estimating wind-induced loads and structural response of tall buildings, a linear, quasi-steady model for multi-directional wind analysis allows a practitioner to simultaneously examine vibrations in along-wind, acrosswind and torsional directions [16]. Multi-directionality is necessary for dynamic analysis because wind load effects are multi-directional. Furthermore, several studies [15, 17] demonstrate that there is a strong correlation between the local building orientation, another factor that influences directionality effect, and the structural performance. 
For a linear elastic building subjected to turbulent wind loads with a mean-wind, horizontal incidence angle $\Psi$, the PSD of the generalized quasi-steady buffeting forces is

$$
S_{Q_{b} Q_{b}}(n)=\iint_{0}^{h} \frac{\rho^{2} D^{2}}{4} \bar{U}\left(z_{1}\right) \bar{U}\left(z_{2}\right) \boldsymbol{\Phi}^{T} \boldsymbol{C}^{\star}\left[\begin{array}{cc}
S_{\mathrm{uu}}\left(n, z_{1}, z_{2}\right) & 0 \\
0 & S_{\mathrm{vv}}\left(n, z_{1}, z_{2}\right)
\end{array}\right] \boldsymbol{C}^{\star, T} \boldsymbol{\Phi} d z_{1} d z_{2}
$$

where

$$
C^{\star}=\left[\begin{array}{ccc}
\cos \Psi & -\sin \Psi & 0 \\
\sin \Psi & \cos \Psi & 0 \\
0 & 0 & 1
\end{array}\right]\left[\begin{array}{cc}
2 C_{D}(\Psi) & C_{D}^{\prime}(\Psi)-C_{L}(\Psi) \\
2 C_{L}(\Psi) & C_{L}^{\prime}(\Psi)+C_{D}(\Psi) \\
2 D C_{M}(\Psi) & D C_{M}^{\prime}(\Psi)
\end{array}\right]
$$

In the previous equations, $S_{Q_{b} Q_{b}}$ is the PSD matrix of the generalized buffeting forces; $\rho$ is the air density; $D$ is a reference floor-plan dimension (usually in the across-wind direction at $\Psi=0 ; \bar{U}(z)$ is the mean wind speed profile evaluated at height $z ; \boldsymbol{\Phi}$ is the mode shape matrix of the structure that can lead to inter-modal coupling arising from non-uniplanar, complex structural modes shapes; $C_{D}(\Psi), C_{L}(\Psi), C_{M}(\Psi)$ is the aerodynamic coefficients of drag force, transverse (lift) force and torque about $z$, respectively; $C_{D}^{\prime}(\Psi), C_{L}^{\prime}(\Psi), C_{M}^{\prime}(\Psi)$ are the corresponding derivatives with respect to $\Psi ; S_{\mathrm{uu}}\left(n, z_{1}, z_{2}\right)$ is the cross spectral density function of the along-wind turbulence component, evaluated at elevations $z_{1}$ and $z_{2}$, and $S_{\mathrm{vv}}\left(n, z_{1}, z_{2}\right)$ is the across-wind turbulence component. The cross-spectral density function between the two turbulence components is omitted due to a weak correlation. Furthermore, a set of dimensionless "model curves" in Eq. (3)-Eq. (4) are utilized to facilitate the parametric study of wind loads, extracted from HFFB wind tunnel tests [18].

$$
\begin{gathered}
\frac{n S_{Q_{x} Q_{x}}(n)}{\left(\frac{1}{2} \rho \bar{U}^{2}(h) D h\right)^{2}}=\frac{a_{D} f}{1+b_{D} f^{c_{D}}} \\
\frac{n S_{Q_{y} Q_{y}}(n)}{\left(\frac{1}{2} \rho \bar{U}^{2}(h) D h\right)^{2}}=\frac{a_{L} f}{1+b_{L} f^{c_{L}}}+d_{L} f^{2} \exp \left[-\left(\frac{f-e_{L}}{f_{L}}\right)^{2}\right]
\end{gathered}
$$

\subsection{Brief introduction to PBWE analysis}

A general procedure for PBWE [19] analysis is summarized in Fig. 1, which describes the key steps that are usually involved in the assessment for structural design. The evaluation of each of the joint probability density functions (PDFs) represents a particular step: $f(I M)$ for site and structure-specific hazard analysis, $f(S P)$ for structural characterization, $f(I P \mid I M, S P)$ for interaction analysis, $f(E D P \mid I M, I P, S P)$ for structural analysis, $f(D M \mid E D P)$ for damage analysis and $f(D V \mid D M)$ for loss analysis. When the performance related to a decision variable $D V$ is expressed by a limit state, which can be associated with an EDP (e.g. the top-floor lateral drift), the procedure of PBWE assumes $D V=E D P$ and can be concisely summarized in Eq. (5) below, where $G(\cdot)$ is a complementary cumulative distribution function (CCDF).

$$
G(E D P)=\iiint G(E D P \mid I M, I P, S P) f(I P \mid I M, S P) f(I M) f(S P) \mathrm{d} I M \mathrm{~d} I P \mathrm{~d} S P
$$




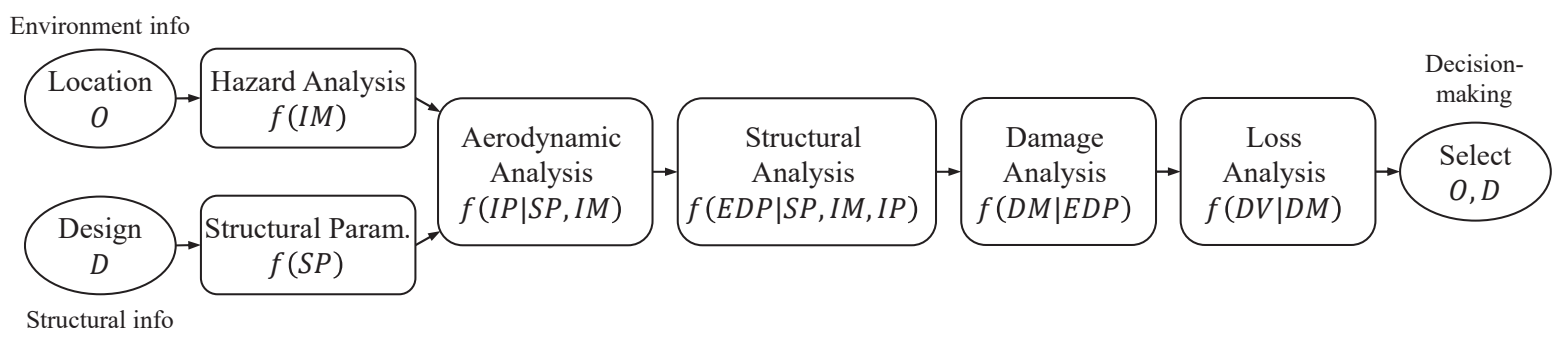

Figure 1: PBWE flowchart, reproduced from [19]. $I M$ : intensity measures; $S P$ : structural parameters; $I P$ : interaction parameters; $E D P$ : engineering demand parameters; $D M$ : damage measures; $D V$ : decision variables.

\section{NOVEL, LSAMC-BASED SIMULATION METHOD}

\subsection{Wind load modeling in mixed wind climates}

A parametric angular-linear distribution model [20] can be employed for the description of mean-wind speed and direction at a reference height (e.g. building's rooftop). Based on the marginal distributions of the two random variables (speed and direction), the general form of the joint PDF is given by Eq. (6), where the two marginal PDFs are combined using a third angular PDF $g(\zeta)$.

$$
f_{V, \Theta}(v, \theta)=2 \pi g(\zeta) f_{V}(v) f_{\Theta}(\theta) \quad 0 \leqslant v<\infty, 0 \leqslant \theta, \zeta<2 \pi
$$

In the previous equation, $g(\zeta)=2 \pi\left[F_{\Theta}(\theta)-F_{V}(v)\right]$ and $F_{\Theta}(\theta), F_{V}(v)$ are the marginal $\mathrm{CDF}$ s of the wind direction and wind speed, respectively. The Gumbel distribution can be used to model the magnitude of wind speeds (annual maxima) for non-hurricane winds, and a Weibull distribution for hurricane winds, specified in Eq. (7) and Eq. (8), respectively. For the corresponding wind direction, a model based on a mixture of von Mises distributions [21], as expressed in Eq. (9) below, enables the inclusion of several prevailing wind directions.

$$
\begin{aligned}
f_{V}(v ; \mu, \gamma) & =\frac{1}{\gamma} \exp \left\{-\left[\frac{v-\mu}{\gamma}+\exp \left(-\frac{v-\mu}{\gamma}\right)\right]\right\} \\
f_{V}(v ; k, \lambda) & =\frac{k}{\lambda}\left(\frac{v}{\lambda}\right)^{k-1} \exp \left[-(v / \lambda)^{k}\right] \\
f_{\Theta}(\theta) & =\sum_{j=1}^{N_{m}} \frac{\omega_{j}}{2 \pi I_{0}\left(\kappa_{j}\right)} \exp \left[\kappa_{j} \cos \left(\theta-\mu_{j}\right)\right]
\end{aligned}
$$

In Eq. (9), $N_{m}$ is the number of components in the mixture model; $\omega_{j}$ are the nonnegative weights for each component that must satisfy the condition $\sum_{j=0}^{N_{m}} \omega_{j}=1 ; I_{0}\left(\kappa_{j}\right)$ is the modified Bessel function of the first kind and order zero, evaluated at a nonnegative quantity $\kappa_{j} ; \mu_{j}$ is the mean directions for each prevailing wind [21].

For a site that is subjected to more than one type of extreme wind events, the wind modeling is better achieved by separately examining each meteorological phenomenon, thus yielding a comprehensive probabilistic description of the local wind environment [22]. This study examines a local wind climate typical of the Mid-Atlantic coast of the United States of America that 
is subjected to both extra-tropical depressions and hurricanes. Therefore, the joint CDF of the annual maxima of the wind speed and direction, is formulated as:

$$
F_{m}(v, \theta)=F_{H}(v, \theta) F_{N H}(v, \theta)
$$

where $f_{m}(v, \theta), f_{H}(v, \theta), f_{N H}(v, \theta)$ are the joint PDFs of the annual maximum mean-wind speed and direction in mixed, hurricane and non-hurricane wind climates, respectively; $f_{H}(v)$, $f_{N H}(v)$ are the marginal PDFs of the magnitude of the maximum mean-wind speeds for hurricane winds and non-hurricane winds; $f_{H}(\theta), f_{N H}(\theta)$ are the marginal PDFs of the corresponding directions, respectively.

$$
\begin{aligned}
f_{m}(v, \theta) & =\frac{\partial^{2} F_{m}(v, \theta)}{\partial v \partial \theta} \\
& =f_{H}(v, \theta) F_{N H}(v, \theta)+f_{H}(v) f_{N H}(\theta)+F_{H}(v, \theta) f_{N H}(v, \theta)+f_{H}(\theta) f_{N H}(v)
\end{aligned}
$$

where $f_{m}(v, \theta), f_{H}(v, \theta), f_{N H}(v, \theta)=$ the joint PDFs of annual maximum wind speed and direction in mixed, hurricane and non-hurricane wind climates, respectively; $f_{H}(v), f_{N H}(v)$ $=$ the marginal PDFs of the magnitude of the maximum speeds for hurricane winds and nonhurricane winds; and $f_{H}(\theta), f_{N H}(\theta)=$ the marginal PDFs of the corresponding directions, respectively.

\subsection{Structural failure probability and LCCA}

The objective of the fragility analysis is to estimate the probability that a specific EDP, conditional on the examined $I M$, exceeds a preselected threshold. Following previous studies, the $E D P$ is chosen as the lateral rooftop drift; the vector $I M$ consists of two random variables, the mean wind speed at the building rooftop, $\bar{U}_{h}$, and the corresponding mean wind direction, $\bar{\theta}$. Structural fragility can be explicitly expressed by Eq. (12), where $d_{j}$ is the preselected threshold for the $j$-th $E D P$. In this study, the threshold for the lateral drift of a building can be set as $1 / 400$ of the building height [23]. Note that the obtained fragility is a fragility surface, allowing for the assessment of the multi-directionality effect induced by the wind.

$$
f_{j}(v, \theta)=P\left(E D P_{j}>d_{j} \mid \bar{U}_{h}=v, \bar{\theta}=\theta\right)
$$

Having obtained the joint PDF of intensity measures $f(I M)$ and the fragility, the structural failure probability with respect to the $j$-th $E D P$ can be estimated by Eq. (13). Finally, the annual probability of $j$-th $E D P$ exceeding the preselected threshold is described by Eq. (14) [24].

$$
\begin{gathered}
P_{j \text {,event }}=\int_{0}^{2 \pi} \int_{0}^{\infty} f_{j}(v, \theta) f_{V, \Theta}(v, \theta) \mathrm{d} v \mathrm{~d} \theta \\
P_{j, \text { annual }}=1-\exp \left(-\nu P_{j, \text { event }}\right)
\end{gathered}
$$

In Eq. (14) $\nu$ is the average annual number of occurrences in the local mixed climate. Assuming independence between hurricane and non-hurricane incidents, $\nu$ is estimated as the summation of the annual rate of hurricane landfall and the number of monthly maximum nonhurricane wind speeds occurring in any one year.

Capitalizing on the quantification of structural failure probability, the LCCA can be implemented to predict damage-induced monetary losses over the structural life-time (with $t$ unit 
time in years). The normalized, expectation of the relative intervention (maintenance, repair, retrofitting, etc.) cost $C_{E}(t)$, adapted from the cost function in [25], can be calculated as:

$$
C_{E}(t)=\mathbf{E}\left[\frac{C(t)-C_{0}}{C_{0}}\right]=\mathbf{E}\left[\sum_{i=1}^{N(t)} \sum_{j=1}^{k} \epsilon_{j} e^{-\lambda t_{i}} P_{j}\right]
$$

In the previous equation, $\mathbf{E}[\cdot]$ denotes the expectation operation; $C(t)$ is the expected total cost induced by damages; $C_{0}$ is the initial construction cost of a structure; $0 \leq t_{i} \leq t$ is the random variable describing the time when the $i$-th hazard occurs; $\epsilon_{j}=C_{j} / C_{0}$ is a normalized relative cost of the $j$-th limit state being violated at time $t_{i}$ calculated as the ratio of the expected intervention cost related to the $j$-th limit state, $C_{j}$, to the initial cost; $\lambda$ is the constant discount rate, which places more weight on hazards occurring closer to the completion date of the structure over those that are not; $P_{j}=P_{j \text {,annual }}$ is the failure probability of the $j$-th limit state, i.e. the probability that intervention relating to the $j$-th limit state is desired; $k$ is the total number of limit states considered; $N(t)$ is the number of wind occurrences, which is a function of $\nu$ during the lifetime.

\subsection{LSAMC method}

Similar to the idea of the Latin Hypercube sampling, the LSAMC method uses the layered sampling of random variables within a Monte-Carlo simulation environment. As proposed by [13], the key steps of the LSAMC method include: (i) dividing the range of each random variable into a finite number of adjacent non-overlapping equally-probable intervals; (ii) constructing a subspace of the random variables for each iteration, consisting of one of the equally-probable intervals for every random variable; (iii) feeding the subspace to the standard Stochastic Approximation algorithm, which has the form of Eq. (16), to find one root of the examined problem subjected to perturbation. Iterating through all the possible subspaces of the original random variables set yields a sequence of roots to the perturbed problem; this allows to approximate the effects of the noises in a structural system through statistical moments derived from the root sequence. By contrast, the "exact" values are determined by a standard "brute-force" Monte-Carlo simulation.

$$
\begin{aligned}
\hat{\boldsymbol{x}}_{k+1} & =\hat{\boldsymbol{x}}_{k}-a_{k} \boldsymbol{Y}_{k} \\
\boldsymbol{Y}_{k} & =\boldsymbol{g}\left(\hat{\boldsymbol{x}}_{k}\right)+\boldsymbol{e}_{k}\left(\hat{\boldsymbol{x}}_{k}\right)
\end{aligned}
$$

In the previous equation, $\hat{x}_{k}$ is the estimate of the root $x$ at the $k$-th iteration; $Y_{k}$ is the noisy measurement at the $k$-th iteration; $\boldsymbol{g}(\cdot)$ is the general vector-valued function from which the root-finding equation $\boldsymbol{g}(\boldsymbol{x})=\mathbf{0}$ is constructed; $\boldsymbol{e}_{k}$ is the noise introduced by the input of the $k$-th iteration; $a_{k}$ is the gain sequence controlling the step size, which is subject to the convergence conditions $\sum_{k=0}^{\infty} a_{k}=\infty$ and $\sum_{k=0}^{\infty} a_{k}^{2}=0$. Eq. (17) utilizes recommended values of the gain sequence [14], where $\alpha$ is a constant belonging to the range $(0.5,1]$ and $a, A$ are a strictly positive constant and a nonnegative constant, respectively.

$$
a_{k}=\frac{a}{(k+1+A)^{\alpha}}
$$

The main advantage of the LSAMC method is the avoidance of time-consuming, computationallyexpensive Monte-Carlo simulations. However, it is worth noting that the advantage could be 


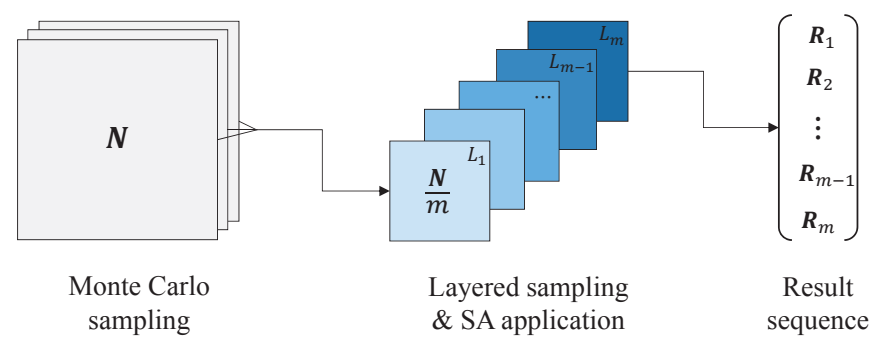

Figure 2: Key steps of the LSAMC method (workflow).

lost if the number of the random variables included were large. To illustrate the potential of the LSAMC method, the/reduction of computing time achieved by the LSAMC method, in the case of two input random variables, can be as large as $88 \%$ compared to brute-force Monte-Carlo simulation for a monopole tower structure under wind loads [13].

\section{NUMERICAL APPLICATION}

In this section, the application of the proposed LSAMC-based simulation to a set of variants of a benchmark tall building is discussed. The LSAMC method is employed for the fragility analysis and the estimation of $P_{j}$ in Eq. (15). Furthermore, dependency of the life-cycle cost variable $C_{E}(t)$ in Eq. (15) on the variable building height is examined.

\subsection{Description of reference building structure and its variants}

Based on the benchmark tall building model of the Commonwealth Advisory Aeronautical Research Council (CAARC), a set of building variants is parametrically constructed. The variants share the same structural parameters (floor plan dimensions, unit mass and mass moment of inertia, etc.) as the CAARC building, except for the building height $h$, which is set to vary as $h \in\{100,150,200,250,300\}$ [m]. The structural properties of the benchmark CAARC building are listed in Table 1.

Table 1: Structural properties of the original benchmark CAARC building.

\begin{tabular}{lll}
\hline Quantity & Unit & Values \\
\hline Height $h_{0}$ & {$[\mathrm{~m}]$} & 183.0 \\
Depth $D$ & {$[\mathrm{~m}]$} & 45.7 \\
Width $B$ & {$[\mathrm{~m}]$} & 30.5 \\
Unit-height mass $m$ & {$[\mathrm{~kg} / \mathrm{m}]$} & $2.23 \times 10^{5}$ \\
Unit-height mass moment of inertia $I_{m}$ & {$\left[\mathrm{kgm}^{2} / \mathrm{m}\right]$} & $5.62 \times 10^{7}$ \\
Lateral-mode fundamental freq. $n_{0, x}, n_{0, y}$ & {$[\mathrm{~Hz}]$} & 0.20 \\
Torsional-mode fundamental freq. $n_{0, z}$ & {$[\mathrm{~Hz}]$} & 0.40 \\
Structural damping ratio $\xi_{0, x}, \xi_{0, y}$ & - & 0.01 \\
\hline
\end{tabular}

For simplification, the fundamental lateral-mode frequencies of the building variants are modifications of the original values, $n_{0, x}$ and $n_{0, y}$ in Table 1 , inversely proportional to the building height, as expressed by Eq. (18) below. The torsional-mode frequency is not modified. In addition, all mode shapes are assumed as dimensionless, monotonic functions, linearly varying 
along the building height.

$$
n_{0, i}^{\prime} \approx \frac{h_{0}}{h} n_{0, i} \quad(i=x, y)
$$

\subsection{Modeling of site wind climate}

The buildings are assumed to be located in Miami, Florida (USA) where hurricanes are frequent. To evaluate the probability distribution of the hurricane wind speed and direction (at landing), a total number of 20019 synthetic hurricane records, simulated in the Miami region over a period of 20000 years [17], are utilized. The angular-linear model for the hurricane wind is obtained by fitting a Weibull model to the magnitudes of the wind speed and a two-component mixture of von Mises model to describe the corresponding directions of the approaching storm; the $g(\zeta)$ function in (6) is modeled as a two-component von Mises mixture model. The resultant joint PDF of the hurricane mean wind speed and direction, evaluated at the rooftop of the original CAARC building with $h=183 \mathrm{~m}$, is illustrated in Fig. 3, which is characterized by two peaks. The predominant peak corresponds to the high probability of winds coming from the east coast and the Atlantic Ocean, whereas the secondary peak is linked to the westerly winds, originating from the Gulf of Mexico and crossing the peninsula.

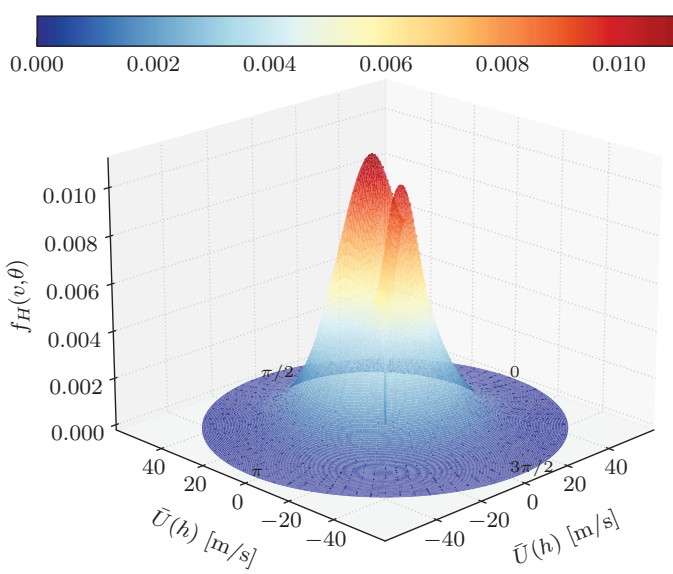

Figure 3: Joint PDF of the mean wind speed and direction for hurricane winds (Miami, Florida, USA) at the reference elevation of the original CAARC building, $h=183 \mathrm{~m}$.

For the non-hurricane winds, the joint PDF of the mean wind speed and direction is obtained by using wind record data collected at an offshore buoy station near the Miami site. The data can be accessed by the National Oceanic and Atmospheric Administration (NOAA) [26]. In order to eliminate the influence of hurricanes, the Atlantic hurricane tracking database, HURDAT2 [27], is used to manually remove the buoy data records related to days when a hurricane approaches within a $500 \mathrm{~km}$ radius of the building site. In particular, the monthly maxima of the gust speed and the corresponding direction are selected from the filtered buoy data records to form a nonhurricane extreme wind dataset. The averaging time interval of the speed is transformed from 3600 seconds to 600 seconds for consistence with hurricane wind data. By fitting a Gumbel distribution to the magnitudes of the wind speeds and likewise a two-component von Mises mixture model to describe the directions, the joint PDF for the non-hurricane wind speed and direction, is illustrated in Fig. 4; quantities are also evaluated at the rooftop of the original 
CAARC building with $h=183 \mathrm{~m}$. Fig. 4 indicates that easterly winds, coming from the sea, play a prominent role in the local non-hurricane climate.

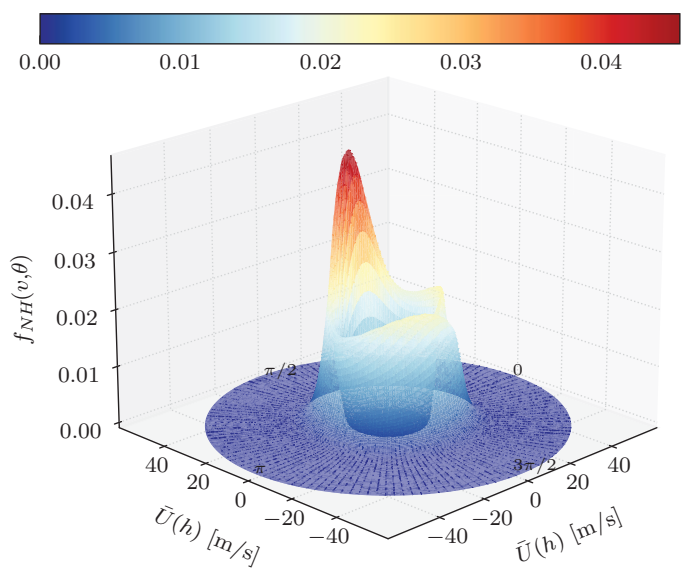

Figure 4: Joint PDF of the mean wind speed and direction for non-hurricane winds (Miami, Florida, USA) at the reference elevation of the original CAARC building, $h=183 \mathrm{~m}$.

The joint PDF of the wind in the mixed climates can be consequently estimated from the Figs. 3 and 4 through Eq. (11). Since an analytical form of the CDF for the von Mises distribution is unavailable, the joint $\operatorname{CDFs}_{H}(v, \theta)$ and $F_{N H}(v, \theta)$ are found through numerical integrations. The joint PDF is plotted in Fig. 5. It is clear that the compound joint PDF combines features of the two separate PDFs related to both hurricane and non-hurricane climate.

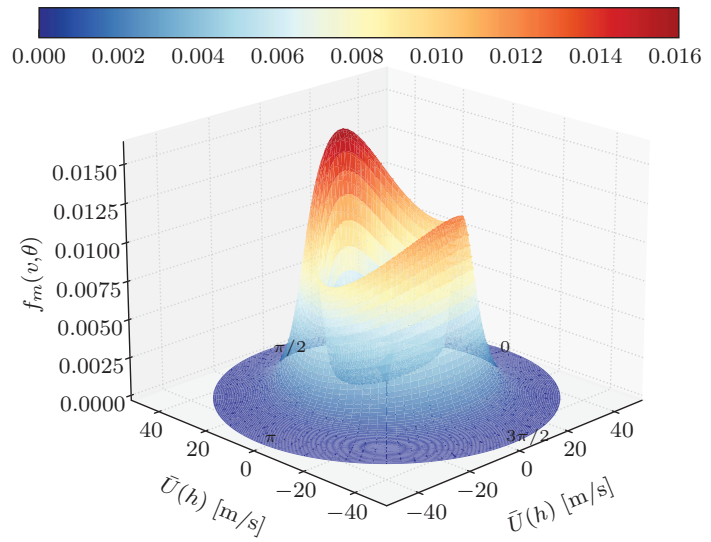

Figure 5: Joint PDF of the mean wind speed and direction for the mixed wind climate of Miami, Florida (USA) at the reference elevation of the original CAARC building, $h=183 \mathrm{~m}$.

\subsection{Characterization of uncertainty in the wind loads}

As an example, this study analyzes uncertainty propagation due to experimental wind load errors by considering a parameter denoted by $\Delta C_{D}$; this quantity describes the deviation of experimentally estimated drag coefficient $C_{D}$ from an average, measured value at a particular incidence angle, i.e., $\Delta C_{D}=C_{D}(\Psi)-\mathbf{E}\left[C_{D}(\Psi)\right]$. Data points of the drag coefficients are extracted from wind tunnel tests on a rigid, scale model of the original CAARC building [18]; 
eleven incidence angles are tested with $\Psi \in\left\{0^{\circ}, 5^{\circ}, 10^{\circ}, 15^{\circ}, 30^{\circ}, 45^{\circ}, 60^{\circ}, 75^{\circ}, 80^{\circ}, 85^{\circ}, 90^{\circ}\right\}$. Experimental data points of $C_{D}$ along with a Fourier series fitting are illustrated in Fig. 6.

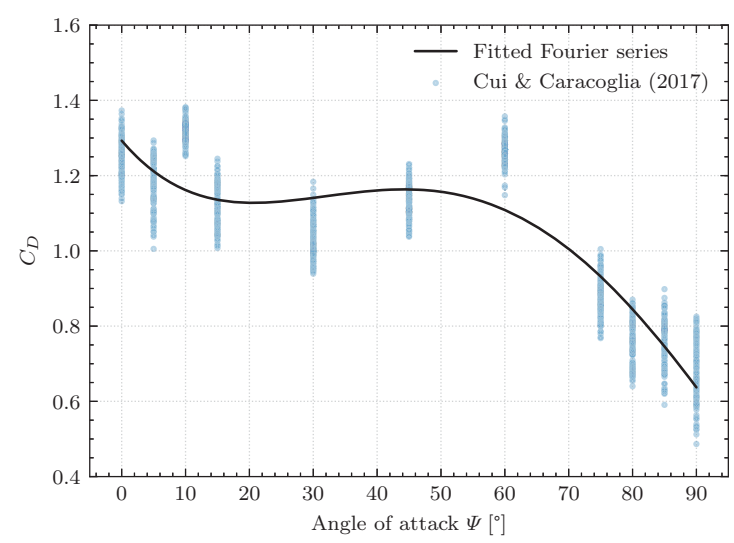

Figure 6: Drag coefficients $C_{D}$ measured at various incidence angles, reproduced from [18].

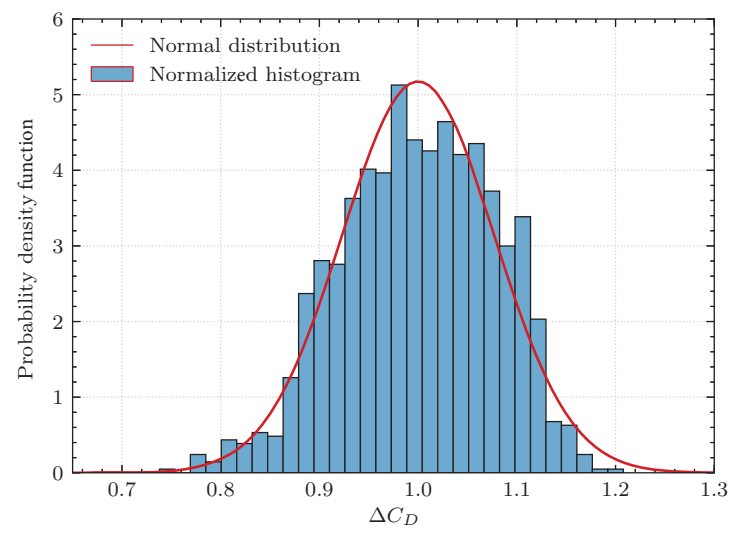

Figure 7: Histogram of the dimensionless uncertainty parameter $\Delta C_{D}$.

Assuming that $\Delta C_{D}$, collected from all the measured data points, is independent of $\Psi$ and that $\Delta C_{D}$ is identically distributed for different angles, a suitable distribution model can be determined through Kolmogorov-Smirnov goodness-of-fit test. A normal distribution, parameterized by a mean value of 1.000 and a standard deviation STD of 0.077 , can adequately describe the empirical histogram (and distribution) of the $\Delta C_{D}$ in Fig. 7.

\subsection{Structural fragility analysis}

Both the standard brute-force Monte-Carlo simulation and the LSAMC method are employed. For the LSAMC method, the number of equally-probable subsets is 10; the parameters of the gain sequence are set as $a=10, A=0, \alpha=0.95$. Structural fragilities against the limit state of rooftop lateral drift are illustrated in Fig. 8; fragility curves related to mean incidence angle $\Psi=\left\{0^{\circ}, 45^{\circ}, 90^{\circ}\right\}$ are exclusively shown for the sake of brevity. The LSAMC results, represented by dashed, non-solid lines, are very close to those found by brute-force Monte-Carlo method plotted as solid lines. By comparing the fragility curves corresponding to different building heights, it is concluded that the wind speed required to reach the prescribed 
performance limit $(h / 400)$ decreases as the building height increases, indicating that a higher building is more sensitive to wind-induced damage as the limit state is probabilistically violated at a lower wind speed.

a)

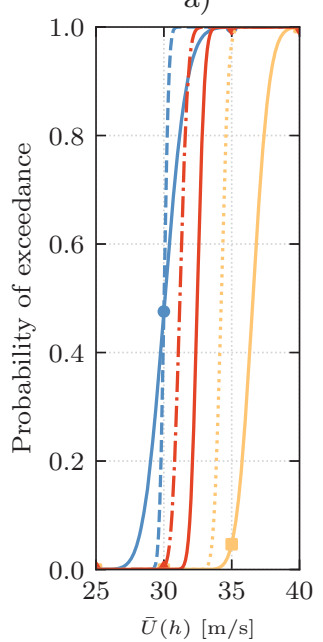

b)

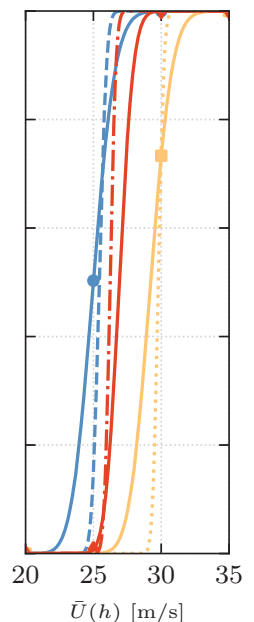

c)

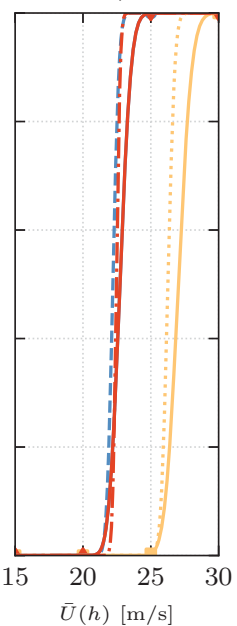

d)

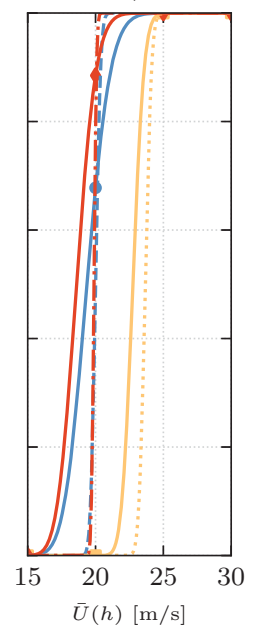

e)

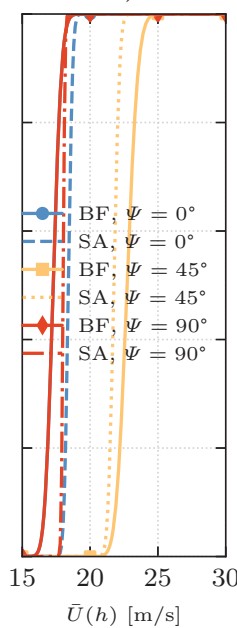

Figure 8: Fragility curves against the limit state of lateral drift with uncertainty parameter $\Delta C_{D}$ : a) $h=100 \mathrm{~m}$, b) $h=150 \mathrm{~m}, \mathrm{c}) h=200 \mathrm{~m}, \mathrm{~d}) h=250 \mathrm{~m}, \mathrm{e}) h=300 \mathrm{~m}$ (BF=brute force, SA=LSAMC).

Fig. 9 depicts the fragility surface of the CAARC building variant of height $h=200 \mathrm{~m}$; other examples of fragility surface for other building variants can be similarly derived and, thus, are not included. The fragility surface is generated by linear interpolation among the fragility curves(Fig. 8) related to the eleven incidence angles. As shown, the fragility surface has an irregular, "ragged" shape, which is attributed to the directionality of the wind load. In Fig. 8c, the curves corresponding to different incident wind directions seem to shift back and forth along the horizontal axis, because the wind-induced force is directional, thus leading to a variation of fragility even for a same performance objective $(E D P)$.

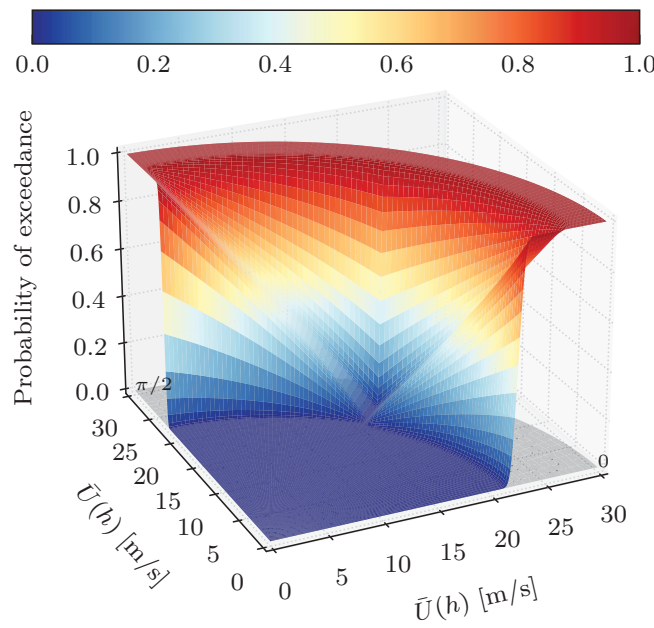

Figure 9: Fragility surface of the CAARC building variant of height $h=200 \mathrm{~m}$. 


\subsection{Expected relative lifetime cost analysis}

Fragility surfaces are used to calculate the failure probability and the expected lifetime cost. According to the Eq. (15), the relative intervention costs due to wind-induced damage, associated with nonstructural failures on the building façade and triggered by disproportionate lateral drift, is $\epsilon=0.02$ and the discount rate is $\lambda=0.05$. Numerical values have been calibrated according to previous studies $[15,17]$. Using the above results of the joint PDF of the mean wind speed and direction in mixed climates and the fragility surfaces, the expected relative lifetime intervention costs due to failures associated with lateral drift are illustrated in Fig. 10. The maximum relative lifetime cost (asymptotic value as $t$ tends to 100 years) is observed for the building variant of height $h=300 \mathrm{~m}$ and is approximately a quarter of the initial cost, while the minimum relative lifetime cost is found for the building with height $h=100 \mathrm{~m}$, which is roughly $2 \%$ of the initial cost. This remark suggests a superlinear relationship between building height and expected relative lifetime cost (i.e. a superlinear increment of $C_{E}(t)$ with height). This behavior can be associated to two aspects. First, due to the wind profile the wind loading on a higher building is generally larger, thus causing larger-magnitude, wind-induced vibrations; second, as discussed in the previous section, a higher building is more slenderer and exhibits greater sensitivity to wind loads of same intensity because of the concurrent decrement of fundamental frequencies. Consequently, the probability of exceeding the lateral drift limit state and damage is superlinearly higher in a taller building, e.g. $h=300 \mathrm{~m}, v s . h=100 \mathrm{~m}$ in Fig. 8.

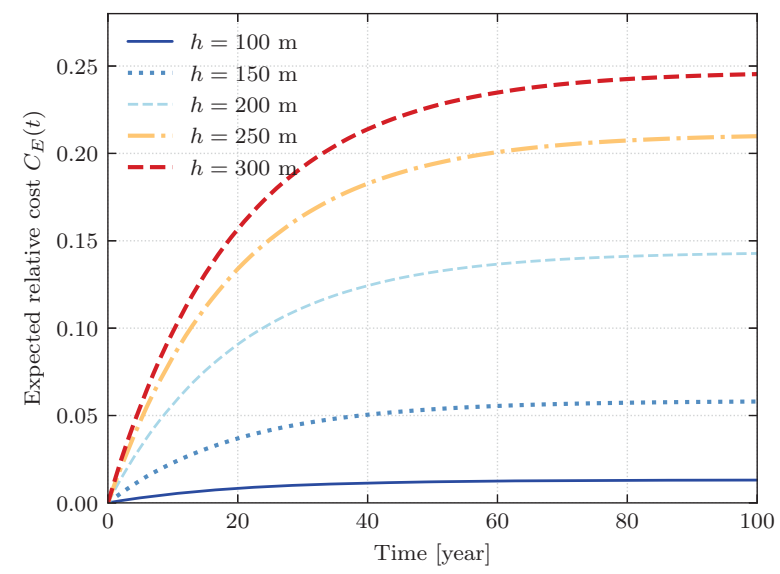

Figure 10: CAARC building variants: expected relative, lifetime intervention cost due to wind-induced damage, associated with nonstructural failures on building facade and triggered by disproportionate lateral drift.

\section{CONCLUSIONS}

This study examined the comprehensive application of the novel LSAMC method in the context of PBWE. Special attention was paid to lifetime intervention cost analysis. A parametric study was conducted, examining a set of variants of the benchmark CAARC building with equal floor-plan dimensions but variable heights. The LSAMC method was used to estimate structural fragilities; results were compared against standard Monte-Carlo simulation. The LSAMC method is adequate for the fragility analysis. The life-cycle cost assessment revealed a superlinear relationship between asymptotic, expected relative cost and building height; this trend can be ascribed to the reduction in structural stiffness and the increase in the sensitivity to wind- 
induced resonant response. This study confirmed the particular significance of nonstructural facade damage caused by wind for operations and maintenance of a high-rise building.

\section{ACKNOWLEDGEMENTS}

This material is based upon work supported in part by the National Science Foundation (NSF) of the United States of America under grant CMMI-1852678 in 2019-2022. Any opinions, findings and conclusions or recommendations are those of the authors and do not necessarily reflect the views of the NSF.

\section{REFERENCES}

[1] A. Kareem, Lateral-torsional motion of tall buildings to wind loads. Journal of Structural Engineering, 111(11), 2479-2496, 1985.

[2] A. Davenport, On the assessment of the reliability of wind loading on low buildings. Journal of Wind Engineering and Industrial Aerodynamics, 11(1-3), 21-37, 1983.

[3] J. D. Holmes, Wind loading of structures: application of probabilistic methods. Progress in Structural Engineering and Materials, 1(2), 193-199, 1998.

[4] F. Minciarelli, M. Gioffrè, M. Grigoriu, E. Simiu, Estimates of extreme wind effects and wind load factors: influence of knowledge uncertainties. Probabilistic engineering mechanics, 16(4), 331-340, 2001.

[5] B. Ellingwood, D. Rosowsky, Y. Li, J. Kim, Fragility assessment of light-frame wood construction subjected to wind and earthquake hazards. Journal of Structural Engineering, ASCE, 130(12), 1921-1930, 2004.

[6] K. C. Kwok, P. A. Hitchcock, M. D. Burton, Perception of vibration and occupant comfort in wind-excited tall buildings. Journal of Wind Engineering and Industrial Aerodynamics, 97(7-8), 368-380, 2009.

[7] E. Bernardini, S. Spence, M. Gioffrè, A. Kareem, A reliability approach for the windinduced response assessment of tall buildings using the high frequency force balance. In 7th International Colloquium on Bluff Bodies Aerodynamics and Applications (BBAA7), pp. 2-6, 2012.

[8] W. Cui, L. Caracoglia, Simulation and analysis of intervention costs due to wind-induced damage on tall buildings. Engineering Structures, 87, 183-197, 2015.

[9] D.-W. Seo, L. Caracoglia, Statistical buffeting response of flexible bridges influenced by errors in aeroelastic loading estimation. Journal of Wind Engineering and Industrial Aerodynamics, 104, 129-140, 2012.

[10] D.-W. Seo, L. Caracoglia, Estimating life-cycle monetary losses due to wind hazards: Fragility analysis of long-span bridges. Engineering Structures, 56, 1593-1606, 2013.

[11] V. Le, L. Caracoglia, Life-cycle cost analysis of a point-like structure subjected to tornadic wind loads. Journal of Structural Engineering, ASCE, 146(2), 04019194, 2020. 
[12] V. Le, L. Caracoglia, A neural network surrogate model for the performance assessment of a vertical structure subjected to non-stationary, tornadic wind loads. Computers \& Structures, 231, 106208, 2020.

[13] G. F. Giaccu, L. Caracoglia, Wind-load fragility analysis of monopole towers by layered Stochastic-Approximation-Monte-Carlo method. Engineering Structures, 174, 462-477, 2018.

[14] J. C. Spall, Introduction to stochastic search and optimization: estimation, simulation, and control, vol. 65. John Wiley \& Sons, 2005.

[15] L. Ierimonti, L. Caracoglia, I. Venanzi, A. L. Materazzi, Investigation on life-cycle damage cost of wind-excited tall buildings considering directionality effects. Journal of Wind Engineering and Industrial Aerodynamics, 171, 207-218, 2017.

[16] W. Cui, L. Caracoglia, A fully-coupled generalized model for multi-directional wind loads on tall buildings: A development of the quasi-steady theory. Journal of Fluids and Structures, 78, 52-68, 2018.

[17] W. Cui, L. Caracoglia, A unified framework for performance-based wind engineering of tall buildings in hurricane-prone regions based on lifetime intervention-cost estimation. Structural Safety, 73, 75-86, 2018.

[18] W. Cui, L. Caracoglia, Examination of experimental variability in HFFB testing of a tall building under multi-directional winds. Journal of Wind Engineering and Industrial Aerodynamics, 171, 34-49, 2017.

[19] M. Ciampoli, F. Petrini, G. Augusti, Performance-based wind engineering: towards a general procedure. Structural Safety, 33(6), 367-378, 2011.

[20] R. A. Johnson, T. E. Wehrly, Some angular-linear distributions and related regression models. Journal of the American Statistical Association, 73(363), 602-606, 1978.

[21] J. A. Carta, P. Ramírez, C. Bueno, A joint probability density function of wind speed and direction for wind energy analysis. Energy Conversion and Management, 49(6), 13091320, 2008.

[22] L. Gomes, B. Vickery, Extreme wind speeds in mixed wind climates. Journal of Wind Engineering and Industrial Aerodynamics, 2(4), 331-344, 1978.

[23] ASCE, Prestandard for Performance-Based Wind Design. American Society of Civil Engineers, Reston, VA, USA, 2019.

[24] P. J. Vickery, P. F. Skerlj, L. A. Twisdale, Simulation of hurricane risk in the U.S. using empirical track model. Journal of Structural Engineering, ASCE, 126(10), 1222-1237, 2000 .

[25] Y.-K. Wen, Y. Kang, Minimum building life-cycle cost design criteria. i: Methodology. Journal of Structural Engineering, 127(3), 330-337, 2001.

[26] NOAA, National Data Buoy Center. http://www.ndbc.noaa.gov/, (accessed April 2, 2020). 
[27] NOAA, National Hurricane Center and Central Pacific Hurricane Center. https:// www. nhc.noaa.gov/, (accessed December 15, 2020). 